\title{
Surgery for glue ear: the English epidemic wanes
}

Nick Black

\begin{abstract}
Objectives - To describe the progress of the epidemic of surgery for glue ear since 1983 and trends in the use of different operative procedures.

Design - Analysis of routine hospital data. Setting - Thirteen health districts in the Oxford and East Anglian regions.

Main measures - Annual rates of surgery in children under 10 years of age.

Results - The rate of surgery for glue ear reached a peak in 1986 since when it has declined by $12 \cdot 6 \%$. The rate peaked in all 13 districts but at different times over a six year period (1984-1989/90). Following the peak, district rates plateaued in eight districts and declined in five. These changes have been accompanied by: an increase in the proportion of operations confined to the tympanic membrane since 1983 (from $40 \%$ to $60 \%$ ); an increase in the use of grommets after myringotomy (from $50 \%$ to $94 \%$ since 1980 ); and an increased use of day surgery for ear-only operations (from about $10 \%$ in the late 1970 s to $50 \%$ in 1987/88).

Conclusions - The previously reported epidemic of surgery for glue ear is waning. This seems to be a result of changes in the clinical judgment of general practitioners and surgeons as to its use and possibly of a reduced demand from parents.
\end{abstract}

( Epidemiol Community Health 1995;49:234-237)

The rate of surgery for children suffering from glue ear (otitis media with effusion) increased considerably in industrialised countries during the 1970s and 1980s. This surgical epidemic is well recognised, although it has not been possible to document it in many countries, including the USA, because of a lack of accurate and complete routine data collection systems. ${ }^{1}$ Previously, it has been shown that the rate of surgery in the UK rose by $74 \%$ between 1975 and 1982 in children living in Oxford health region $^{2}$ and by more than $100 \%$ for those living in East Anglian region. ${ }^{3}$ Similar trends were subsequently reported for the Tayside region of Scotland. ${ }^{4}$

During the 1980s the appropriateness of surgery for glue ear was increasingly questioned in the light of the results both of observational studies and randomised controlled trials. ${ }^{5}$ This led to the development of guidelines in some countries that advocated a more conservative use of surgery. ${ }^{67}$ But meanwhile nothing is known about what has been happening to the epidemic over the past decade. This paper describes trends in the surgical rate in two
English health regions and changes in the operative procedures used.

\section{Methods}

Annual numbers of surgical operations for glue ear performed in NHS hospitals in the Oxford and East Anglian health regions were obtained for 1975 to $1990 / 91$. The data distinguished the specific procedures used from 1976 onwards. Data came from the Hospital Activity Analysis (HAA) system up until 1986 and then from the District Information System. Cases were included if they were aged under 10 years and had undergone myringotomy or grommet insertion with or without adenoidectomy with or without tonsillectomy.

From 1975-1987/88 procedures were coded according to the OPCS 3rd revision (193.1193.4 ; $193 \cdot 1-193.4$ with 235 ; $193 \cdot 1-193.4$ with 233) and from $1988 / 89$ the OPCS 4 th revision (D15.1-15.4, 15.8, 15.9; D15.1-15.4, $15 \cdot 8,15 \cdot 9$ with E20; D15.1-15.4, 15.8, 15.9 with E20 and F34). Care was taken to avoid double counting when surgery was bilateral. Data for Oxford region distinguished between myringotomy and grommet insertion and data for East Anglian region up until 1987/88 distinguished between inpatients and day cases.

Annual intervention rates were calculated for all the health districts that existed in 1975 except Aylesbury Vale which did not provide HAA data at that time. For assessing longitudinal trends in district rates, changes in boundaries were ignored. Thus, Huntingdon has continued to be included with Cambridgeshire. Numerators were based on the number of operations performed within a district and denominators were the resident population aged under 10 years.

Adjustments were made in all 13 districts for shortfalls in the clinical coding in otolaryngology. Of the $195(13 \times 15)$ annual rates, clinical coding was over $90 \%$ complete in 151 $(77 \cdot 4 \%)$ and over $98 \%$ complete in $59(30 \cdot 3 \%)$. It was assumed that failure to code procedures was not influenced by the procedure carried out. Intervention rates for surgery for glue ear were therefore adjusted according to the overall shortfall for the specialty.

Information on the number of children treated in the nine independent hospitals in the two regions were obtained for 1992 to assess the extent to which any changes in the rate of cases in NHS hospitals might have been counteracted by activity outside the NHS.

The total annual number of cases in the study population (adjusted for coding shortfalls) varied between 3272 and 7214. Secular trends in the rate of surgery for the total study population (505 700-611 000) and for each of 


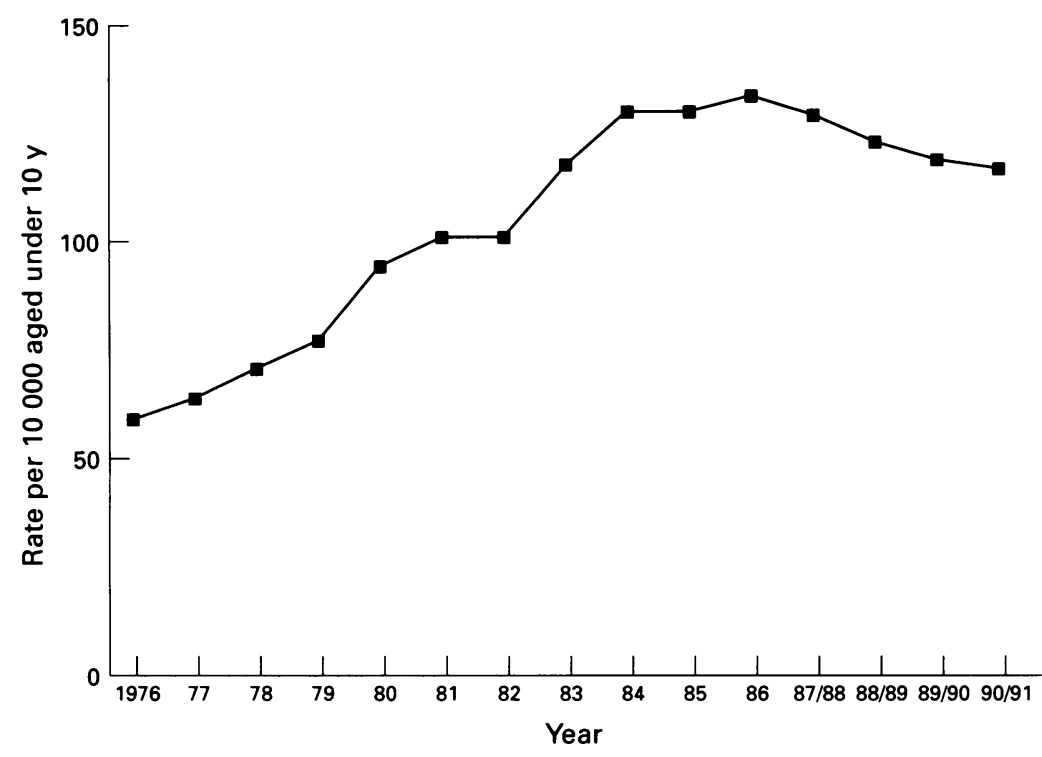

Figure 1 Rates of surgery for glue ear in the Oxford and East Anglia health regions.

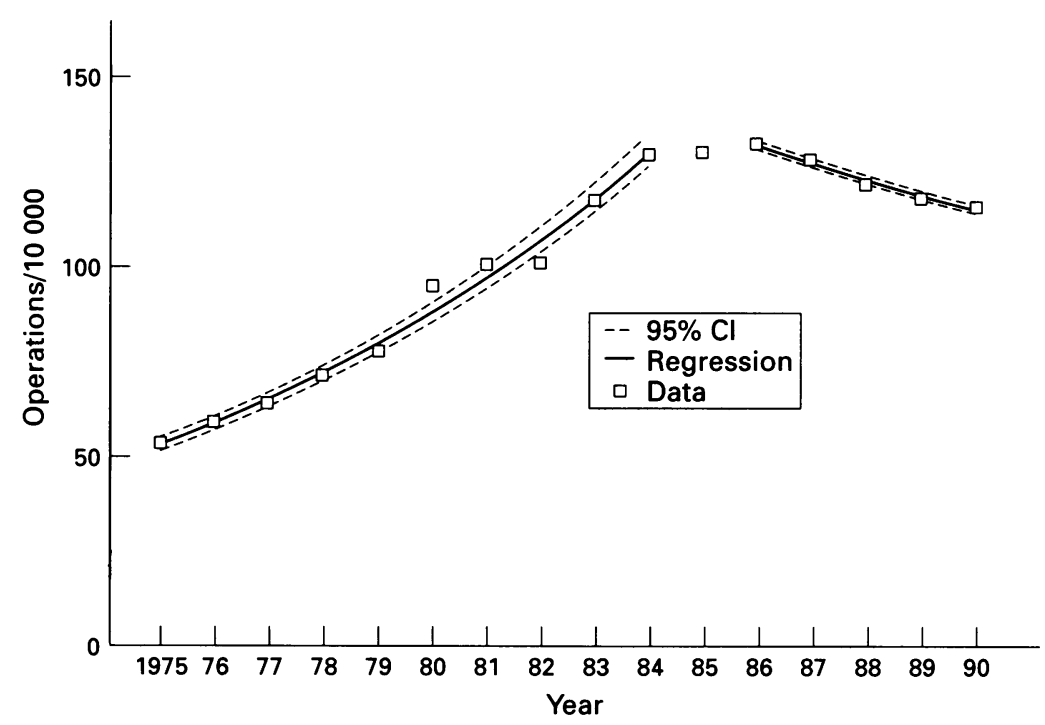

Figure 2 Operations for glue ear in the Oxford and East Anglia health regions, 1975-90.

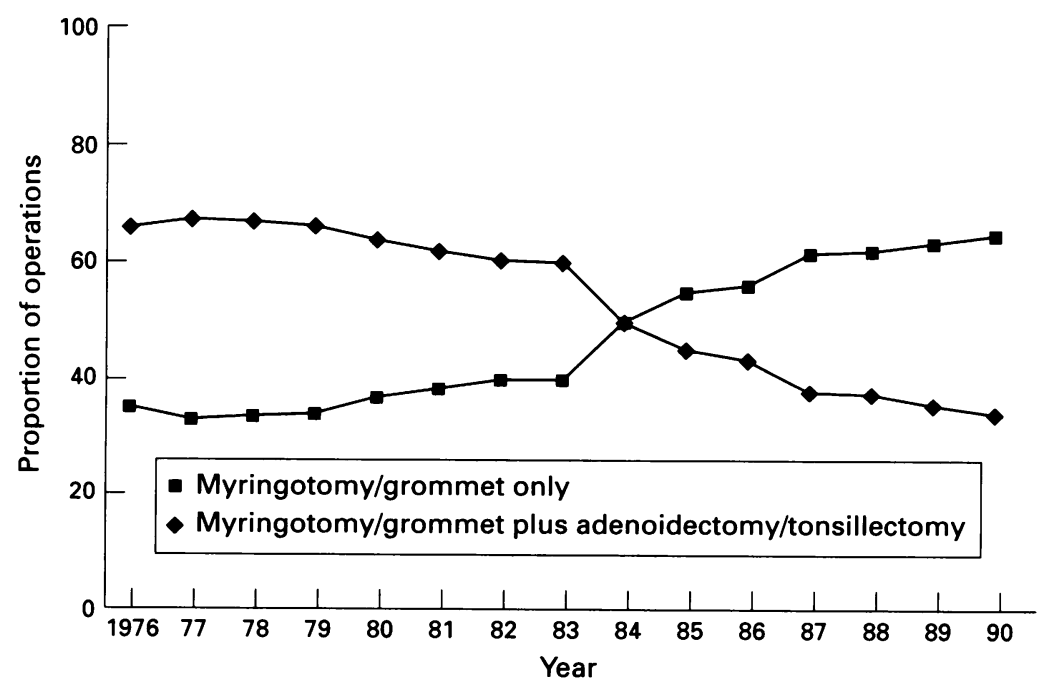

Figure 3 Trends in the proportions of ear operations that included adenoid/tonsil surgery. the 13 constituent districts were examined. Changes in the trends in the rate of surgery were assessed by fitting regression lines based on $\log$ transformation of the actual rate. Autocorrelation was checked for using the DurbinWatson statistic. This sought to establish that the chance of any annual rate being above or below the computed regression value was 50: 50. Evidence of auto-correlation would suggest an important independent variable had been left out of the regression analysis. Trends in the use of different surgical procedures and use of day care were also described.

\section{Results}

RATE OF SURGERY

The rate of surgery in the study population continued to rise during the mid-1980s, reached a peak in 1986, but has been falling since then (fig 1). Between 1986 and 1990/91 the rate fell by $12 \cdot 6 \%$ from 131.8 to $115 \cdot 2$ per 10000 . The extent of the decline in the rate was similar in both health regions: a fall since 1986 of $15.0 \%$ in East Anglia and, since 1984, of $16.6 \%$ in Oxford. There was no significant auto-correlation (Durbin-Watson statistic: $1975-84=2 \cdot 13 ; 1986-90 / 91=2 \cdot 15)$. The slopes of the regression lines $(1975-84=10 \cdot 4 \%$ per annum (95\% CI 9.6, 11.2); 1986-90/91= $-3.4 \%$ per annum $(95 \%$ CI $-3.0,-3.9)$ ) were significantly different (fig 2 ).

The rate had peaked in all 13 districts by 1989/90 but this occurred at different times: 1984 Oxfordshire, Northampton, Wycombe; 1985 Norwich, East Berkshire; 1986 Great Yarmouth, West Norfolk, West Berkshire; 1987/88 Peterborough, Cambridgeshire, Kettering; 1988/89 West Suffolk; 1989/90 East Suffolk. Two distinct trends occurred after a district's peak year - in eight districts the rate of surgery remained at the peak level while in the other five the rate declined after the peak year.

During 1992, 725 children were operated on for glue ear in the nine local independent hospitals.

PROCEDURES USED

There has been a trend away from treatments that include adenoidectomy (with or without tonsillectomy) towards operations which involve only the tympanic membrane - a myringotomy usually with a grommet insertion (fig 3). Until 1983, about $60 \%$ of operations included surgery on the adenoids or tonsils and only $40 \%$ were restricted to the tympanic membrane. By 1987 these proportions had reversed. While this change occurred in all 13 constituent districts, the extent of the change varied so that the proportion of ear-only operations carried out in 1990/91 ranged from $12 \%$ to $50 \%$ between districts.

There has also been a trend towards inserting grommets when a myringotomy is performed (fig 4). In the late 1970 s, grommets were inserted in only about half of all myringotomies performed in Oxford region. The proportion steadily increased, reaching $94 \%$ by $1990 / 91$. 


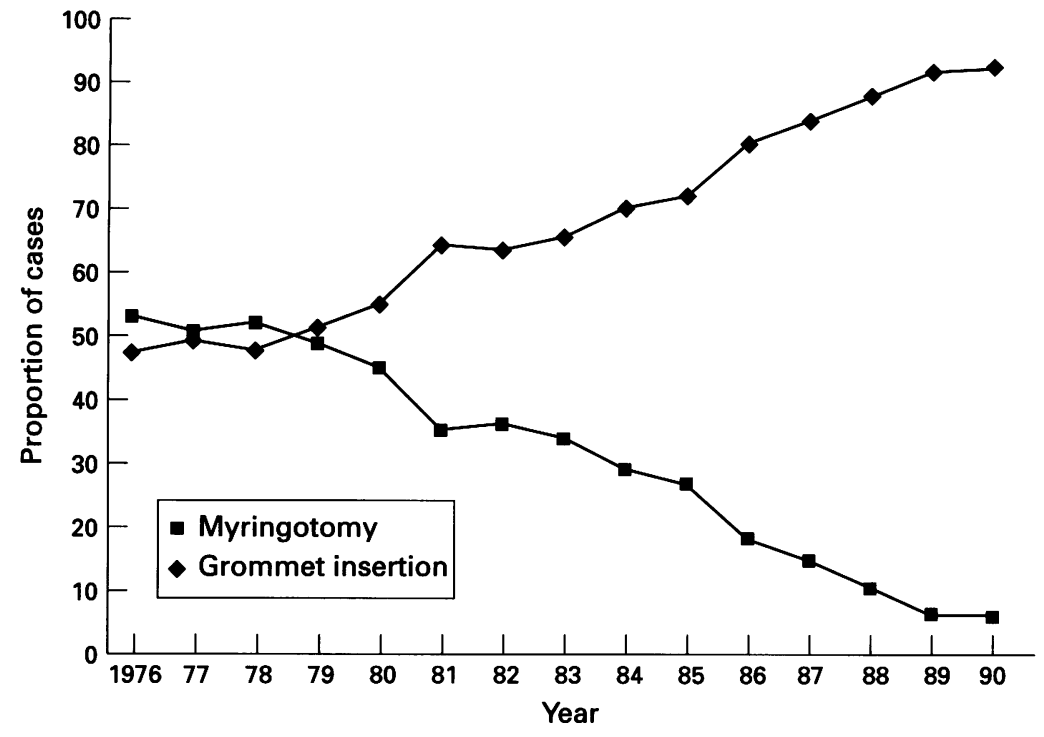

Figure 4 Proportion of ear operations in which grommets were inserted, Oxford health region.

At the same time, the amount of interdistrict variation in the use of grommets fell. In 1976 the proportion of myringotomies in which a grommet had been inserted varied between districts from $12 \%$ to $73 \%$. By $1990 / 91$ it varied only from $90 \%$ to $97 \%$.

USE OF DAY SURGERY

Day surgery has become increasingly common for children in whom surgery is confined to the tympanic membrane (fig 5). In the late 1970s only about $15 \%$ of these cases were treated on a day basis. This had risen to around $50 \%$ by $1987 / 88$, though its use still varied considerably between districts, from $5 \%$ to about $60 \%$.

\section{Discussion}

The increasing rate of surgery for glue ear reported in the early 1980 s peaked in the mid 1980 s since when the epidemic has been wan-

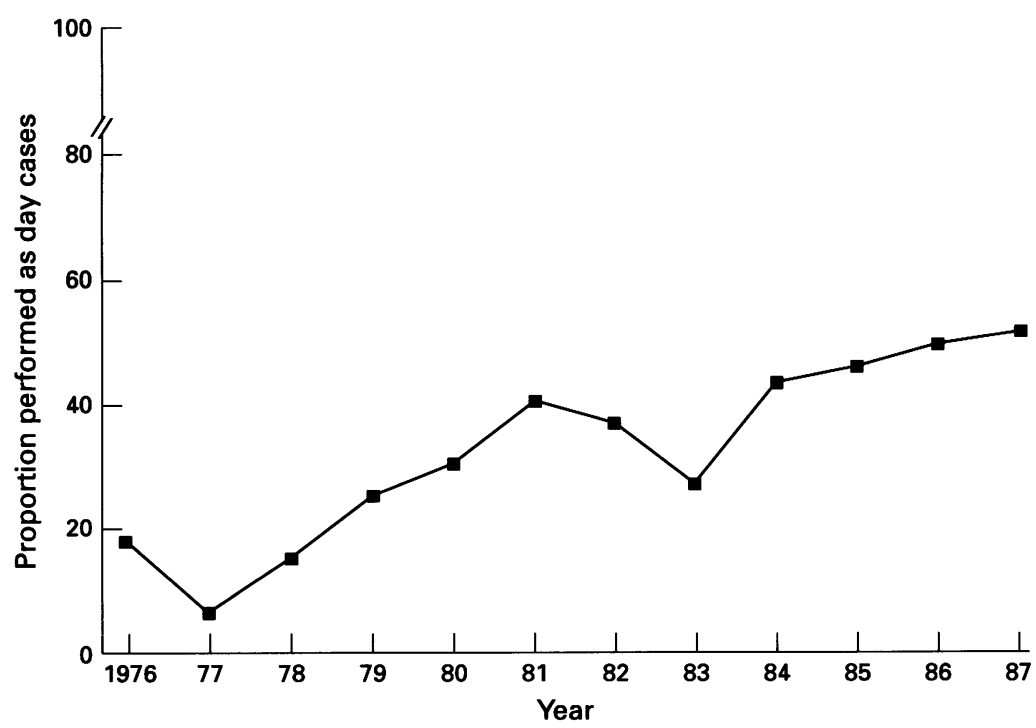

Figure 5 Proportion of ear-only operations performed as day cases in the East Anglian health region.
Possible explanations for the waning of the epidemic of surgery for glue ear

Statistical artifacts

- increasing shortfalls in clinical coding of surgery for glue ear relative to other otolaryngological procedures

increasing migration of surgical cases out of the regions

- increasing surgery in independent hospitals

Demand factors

- lowering of parental expectations

- fall in morbidity (glue ear prevalence)

Supply factors

- reduction in availability of otolaryngological services

- reduction in referral rate from general practitioners

- adoption of more stringent criteria for surgery by otolaryngologists

ing. The peak occurred in different health districts at different times over a six year period (1984-1989/90). After the peak, district rates have either plateaued or declined. The waning of the epidemic has been accompanied by three appreciable procedural changes - an increase in the proportion of operations confined to the tympanic membrane; an increased use of grommets after myringotomy; and an increased use of day surgery for children undergoing ear surgery only.

Before discussing possible reasons for the waning of the epidemic (table), some of the assumptions about the routine data used need to be considered. Firstly, shortfalls in clinical coding of surgery for glue ear were assumed to reflect the overall shortfall in otolaryngology. Given that for most districts and for the study population as a whole the shortfall was less than $10 \%$, this assumption is unlikely to have introduced any serious error.

Secondly, the calculation of district rates of surgery assumed that the size of the catchment populations of the participating hospitals were similar to the resident populations of the districts in which they were located. In other words, most children would have been treated in the hospital located in their health district. While major cross boundary flow between districts would affect the absolute district rate, it would neither affect the rate for the whole study population nor trends in district rates unless such flows changed over time (which is not thought to have happened).

Thirdly, the exclusion of data from independent hospitals will have underestimated the true rate of surgery. Estimates from the only published studies ${ }^{89}$ suggest that in 1981 independent hospitals may have contributed an additional $15 \%$ and in 1986, an extra $27 \%$ (Jon Nicholl - personal communication). However, data obtained directly from the independent hospitals in the two regions in 1992 showed that they contributed an additional $11 \%$ to the number carried out in NHS hospitals. Thus, even if no operations were performed in independent hospitals in 1986, there would still have been a reduction in the overall surgical rate over the ensuing years.

Fourthly, the model used to fit the regression lines deliberately excluded data for 1985 because the turning point in the direction of the trends occurred between 1984 and 1986. Similar results were obtained when all three years (1984-86) were eliminated.

The final methodological concern is that the two regions studied are unrepresentative of the 
UK. Between 1975 and 1980 Oxford had the highest and East Anglia the fourth highest rate of eight regions studied. ${ }^{3}$ This suggests that these two health regions have been in the vanguard of developments in glue ear surgery and this may still have been so in the late 1980 s. If this is true, the waning of the epidemic may not yet be apparent in other regions of the country.

Given that the trends observed are not a statistical artifact, the epidemic in surgery for glue ear may have waned as a result of a fall either in the demand for or supply of operations. A reduction in demand for surgery may have arisen, not because of a fall in morbidity for which there is no evidence, but because of lower parental expectations. While there is some evidence from mass media that lay views of surgery for glue ear have become more critical, it seems unlikely that parental questioning of surgical advice could have made more than a small contribution to reversing the epidemic.

The alternative explanation is a reduction in the supply of surgery. Throughout the period studied the availability of services, as judged by the number of consultant and junior otolaryngologists, has risen, though only insignificantly (Personal communications - Nick Wareham (East Anglia Regional Health Authority) and Marion Davis (Oxford Regional Health Authority)).

The other supply factor to be considered is clinical judgement. In other words, the fall in the surgical rate reflects a change in the opinion of surgeons about the appropriate indications for operating. Differences in clinical judgement over which procedures to use have been a feature of surgery for glue ear for many years. The findings of a recent survey of consultants' views of the clinical management of glue ear ${ }^{10}$ found a level of disagreement similar to that reported in $1977 .{ }^{11}$ The current study has shown that wide variations still exist between districts in the use of adenoidectomy (12-50\%) and day surgery for ear-only operations (5$60 \%)$. The one exception is the insertion of grommets in myringotomies (fig 4). Several trials have shown the ineffectiveness of myringotomies without grommet insertion ${ }^{12-14}$ and these have contributed to the emergence of a clear consensus.

However, changes in surgeons' views as to how to treat glue ear would not of themselves necessarily effect the overall intervention rate if they were still subjected to a high rate of referrals from general practitioners. Thus, a fall in the surgical rate might also require that general practitioners are more selective in their referrals. There is some evidence that this might have happened. While it is no surprise to find concerns expressed by a general practitioner, ${ }^{15}$ leading figures in otolaryngology have in- creasingly questioned the wisdom of contemporary clinical practice. ${ }^{16-18}$ These views, often differing from those expressed previously by the same authors, may reflect the impact of the 10 randomised controlled trials published between 1984 and 1990 (compared with only seven published in the preceding 16 years). ${ }^{5}$

The waning of the epidemic should come as no surprise. Most health care technologies go through a diffusion cycle in which, after a period of adoption, widespread use with overenthusiastic application follows, before a period of more appropriate use occurs in which more stringent criteria are adopted. What we may be witnessing is the start of this final phase. If this turns out to be so, then this, the third era of surgery for glue ear, will be mirroring the time path of the previous two. ${ }^{19}$

Thanks to Sandra Edwards (Oxford RHA) for advice on data quality and to her, Stephen Pearce, Kate Tennyson, Tony Peeke and other statistical and information staff of Oxford RHA and Isabel Moden and David Davies of East Anglian RHA who have supplied data over the past 15 years. Thanks also to the nine independent hospitals in the two regions for supplying or providing access to data. I am indebted to Colin Sanders who carried out the regression analysis.

1 Derkay CS. Pediatric otolaryngology procedures in the United States: 1977-1987. Int F Ped Otorhinolaryngol 1993 25:1-12.

2 Black N. Surgery for glue ear - a modern epidemic. Lancet $1984 ; \mathrm{i}: 835-7$.

3 Black N. Geographical variations in use of surgery for glue ear. F Roy Soc Med 1985;78:641-8.

4 Padgham N, Mills R, Christmas $H$. Has the increasing use of grommets influenced the frequency of surgery for cholesteatoma? f Laryngol Otol 1989;103:1034-5.

5 Stephenson $H$, Haggard M. Rationale and design of surgical trials for otitis media with effusion. Clin Otolaryngol 1992; 17:67-78.

6 Danish Medical Research Council. Secretoric otitis media (glue ear). Consensus report. Copenhagen: Danish Medical Research Council, 1987.

7 Effective Health Care. The surgical treatment of glue ear (otitis media with effusion) in children. Leeds: University of Leeds, 1992. (Bulletin No 4)

8 Nicholl JP, Thomas KJ, Williams BT, Knowledon J. Contribution of the private sector to elective surgery in England tribution of the private sector to elect

9 Nicholl JP, Beeby NR, Williams BT. Role of the private sector in elective surgery in England and Wales, 1986

10 Smith IM, Maw AR. Secretory otitis media: a review of management by consultant otolaryngologists. Clin Otolaryngol 1991;16:266-70.

11 Hibbert J. The current status of adenoidectomy: a survey among otolaryngologists. Clin Otolaryngol 1977;2:239-47.

12 Archard JC. The place of myringotomy in the managemen of secretory otitis media in children. $₹$ Laryngol Otol 1967 81:309-15.

13 Mandel EM, Bluestone CD, Paradise JL, et al. Efficacy of myringotomy with and without tympanostomy tube insertion in the treatment of chronic otitis media with effusion in infants and children: results for the first year of a randomised clinical trial. In: Lim DJ, Bluestone CD Klein JO, et al eds. Recent advances in otitis media with effusion. Philadelphia: Decker, 1984;308-12.

14 Gates GA, Avery CA, Prihoda TJ, et al. Effectiveness of adenoidectomy and tympanostomy tubes in the treatmen of chronic otitis media with effusion. $N$ Engl $F$ Med 1987 317:1444-51.

15 De Melker RA. Treating persistent glue ear in children. $B M \Im$ 1993;306:5-6.

16 Smyth GDL. Toynbee memorial lecture 1992: Facts and fantasies in modern otology: the ear doctor's dilemma. $f$ Laryngol Otol 1992;106:591-6.

17 Maw AR. Using typanometry to detect glue ear in general practice. BMF 1992;304:67-8.

18 Brown HM. Glue ear guidelines (letter). BMF 1993;341: 57.

19 Black NA. Fashion, science and technical change: the history of the treatment of glue ear. Clin Otolaryngol 1985;10: $31-41$. 